\title{
Alternative Confidence Intervals on the Sum of Variance Components in a Simple Regression Model with Unbalanced Nested Error Structure
}

\author{
Dong Joon Park ${ }^{1)}$ and Soo Jin Lee ${ }^{2)}$
}

\begin{abstract}
In order to construct confidence intervals on the sum of variance components in a simple regression model with unbalanced nested error structure, alternative confidence intervals using Graybill and Wang(1980) and generalized inference concept introduced by Tsui and Weerahandi(1989) are proposed. Computer simulation programmed by SAS/IML is performed to compare the simulated confidence coefficients and average interval lengths of the proposed confidence intervals. A numerical example is provided to demonstrate the confidence intervals and to show consistency between the example and simulation results.
\end{abstract}

Keywords : variance components, interval estimation, generalized pivotal quantity

\section{1. 불균형 중첩 오차구조를 갖는 단순 선형 회귀모형}

통계 연구자들이 자료를 수집하는 과정에서 흔히 만나게 되는 불균형 중첩 오차구조를 갖는 단 순 선형 회귀모형은 아래와 같이 쓸 때

$$
\begin{gathered}
Y_{i j}=\mu+\beta X_{i j}+A_{i}+E_{i j} \\
i=1, \ldots, I ; j=1, \ldots, J_{i}
\end{gathered}
$$

여기서, $Y_{i j}$ 는 $i$ 번째 수준의 $j$ 번째 반응변수값, $\mu$ 와 $\beta$ 는 미지의 상수, $X_{i j}$ 는 $i$ 번째 수준의 $j$ 번째 예측변수이고, $A_{i}$ 는 군(cluster)과 관련된 오차항이고 $E_{i j}$ 는 군내의 개별자료와 관련된 오차항으 로서 $A_{i}$ 와 $E_{i j}$ 는 평균이 0 이고 분산이 각각 $\sigma_{A}^{2}$ 과 $\sigma_{E}^{2}$ 인 서로 독립인 정규 확률변수이다. 군의 수 는 $I \geqq 2$ 이고 군의 크기는 $J_{i} \geqq 1$ 으로서 적어도 하나의 $i$ 에 대해 $J_{i}>1$ 이다. 연구자들은 일반적 으로 각 오차항에 대한 분산의 변이성(variability)과 분산의 비율에 관심을 갖는다. 그러나 그에

1) 608-737 Busan Nam-Gu Daeyeon 3-Dong 599-1 Pukyong National University College of Natural Sciences Statistics Major Associate Professor.

E-mail : djpark@pknu.ac.kr

2) 608-737 Busan Nam-Gu Daeyeon 3-Dong 599-1 Pukyong National University College of Natural Sciences Statistics Major Graduate Student. 
88 Dong Joon Park and Soo Jin Lee

못지않게 Smith(1936)와 Satterthwaite(1941, 1946)로부터 시작하여 분산의 선형결합 또한 많은 연구가 되어 왔다(Burdick and Graybill(1992)). 예를 들면 모형 (1.1)에서 반응변수의 분산을 구 하기 위해서는 두 분산 $\sigma_{A}^{2}$ 과 $\sigma_{E}^{2}$ 의 합의 계산이 필요하다. 우리는 2절에서 모형(1.1)에서 나타나 는 두 분산의 합 $\gamma=\sigma_{A}^{2}+\sigma_{E}^{2}$ 에 대한 여러 가지 신뢰구간을 제안한 다음, 3 절에서 SAS/IML로 모 의실헙을 실행하여 신뢰계수와 신뢰구간의 평균길이를 비교함으로써 군의 수의 변화에 따라 어 느 방법이 적절한 신뢰구간을 구축하는지 살펴본다. 그리고 4절에서 실제 예제에 적용하여 신뢰 구간을 구한 다음, 모의실험의 결과와 실제 예제의 결과가 일관성이 있는지 확인하고 5 절에서 결론을 맺는다.

\section{2. 분산의 합에 대한 신뢰구간}

모형 (1.1)을 행렬의 형태로 고쳐 쓰면 다음과 같다.

$$
y=X \underline{\alpha}+B u+e
$$

여기서, $y$ 는 $J \times 1$ 관찰값 벡터, $X$ 는 첫 번째 열의 값이 1 이고 $X_{i j}$ 값들이 두 번째 열을 구성하 는 $J \times 2$ 행렬, $\underline{\alpha}$ 는 모수인 $\mu$ 와 $\beta$ 를 원소로 하는 $2 \times 1$ 벡터, $B$ 는 0 과 1 의 값을 갖는 $J \times I$ 설 계행렬 즉, $B=\oplus_{i=1}^{I} \underline{1}_{J_{i} \times 1}, u$ 는 랜덤효과를 나타내는 $A_{i}$ 를 원소로 갖는 $I \times 1$ 벡터, $e$ 는 랜덤오차 항인 $E_{i j}$ 를 원소로 갖는 $J_{.} \times 1$ 벡터이고 $J_{.}=\Sigma J_{i}$ 이다.

Olsen et al.(1976), Eubank et al.(2001)과 El-Bassiouni(1994)가 제안한 제곱합을 이용하여 Park과 Burdick(2003)은 근사적으로 $\chi^{2}$ 분포를 따르는 아래의 통계량을 제안했다. 즉, 행렬 $W$ 를 $W=F B B^{\prime} F$ 라고 쓸 때, 여기서, $F=X^{*}\left(X^{*} X^{*}\right)^{+} X^{* \prime}-X\left(X^{\prime} X\right)^{+} X^{\prime}$ 이고, $X^{*}=\left[X, B B^{\prime}\right]$ 이고, + 는 Moore-Penrose 역행렬이다. 그리고 $l=1,2, \ldots, m$ 일 때 행렬 $W$ 의 서로 다른 고유값을 $d_{1}, d_{2}, \ldots, d_{m}$ 이라 하고, $r_{l}$ 을 $d_{l}$ 의 개수(multiplicity)라 하면 $\operatorname{rank}(W)=\Sigma r_{l}=I-1=n_{1}$ 임을 보였다. 벡터 $z$ 를 $z=F y$ 로 정의하면 행렬 $W$ 와 벡터 $z$ 를 이용한 평균제곱을 $S_{M}^{2}=\left[z^{\prime} W^{+} z\right] / n_{1}$ 이라 쓰고, 모 형. (1.1)의 분산분석과정에서 나타나는 오차 평균제곱 $S_{E}^{2}$ 은 $S_{E}^{2}=y^{\prime}\left[D_{J}-X^{*}\left(X^{*} X^{*}\right)^{-} X^{*}\right] \boldsymbol{y} / n_{2}$ 로 쓰며 여기서 $n_{2}=J-I-1$ 이다. 그리고 Eubank et al.으로부터 다음이 보여졌다.

$$
\begin{gathered}
\frac{n_{2} S_{E}^{2}}{\sigma_{E}^{2}} \sim \chi_{n_{2}}^{2} \\
\frac{n_{1} S_{M}^{2}}{\sigma_{A}^{2}+\frac{1}{h} \sigma_{E}^{2}} \approx \chi_{n_{1}}^{2} \text { 이고 } \sigma_{E}^{2}=0 \text { 이면 } \frac{n_{1} S_{M}^{2}}{\sigma_{A}^{2}} \sim \chi_{n_{1}}^{2} \\
S_{M}^{2} \text { 과 } S_{E}^{2} \text { 은 서로 독립 } \\
E\left(S_{M}^{2}\right)=\sigma_{A}^{2}+\frac{1}{h} \sigma_{E}^{2}
\end{gathered}
$$

여기서, $h$ 는 행렬 $W$ 의 고유값들의 조화평균이다. 두 분산의 합 $\gamma=\sigma_{A}^{2}+\sigma_{E}^{2}$ 에 대한 신뢰구간을 
구하기 위하여 기대 평균제곱을 다음과 같이 쓴다.

$$
\begin{aligned}
& E\left(S_{M}^{2}\right)=\sigma_{A}^{2}+\frac{1}{h} \sigma_{E}^{2}=\theta_{M} \\
& E\left(S_{E}^{2}\right)=\quad \sigma_{E}^{2}=\theta_{E}
\end{aligned}
$$

그러면 , 식 (2.3)으로부터 $\gamma$ 는 $\gamma=\sigma_{A}^{2}+\sigma_{E}^{2}=\theta_{M}+\left(1-\frac{1}{h}\right) \theta_{E}$ 로 쓸 수 있다.

Park(2003)은 $\gamma$ 에 대한 신뢰구간을 구하기 위하여 (2.4)의 Ting et al.(1990)방법과 Tsui and Weerahandi(1989)가 제안한 일반화 $p$ 값을 활용한 일반화 축량 방법을 이용한 (2.6)을 제안하였 다. 구체적으로 설명하면 (2.2)와 같은 성질을 갖는 $S_{M}^{2}$ 과 $S_{E}^{2}$ 을 서로 독립인 $\chi^{2}$ 확률변수들을 요 구하는 Ting et al.(1990)방법에 적용한 결과 $\gamma$ 에 대한 $100(1-\alpha) \%$ 양쪽 신뢰구간은 다음 식 으로 구해진다.

$$
\begin{gathered}
{\left[S_{M}^{2}+\left(1-\frac{1}{h}\right) S_{E}^{2}-\left\{K_{1}^{2} S_{M}^{4}+\left(1-\frac{1}{h}\right)^{2} K_{2}^{2} S_{E}^{4}+\left(1-\frac{1}{h}\right)^{2} K_{12} S_{M}^{2} S_{E}^{2}\right\}^{\frac{1}{2}}\right.} \\
\left.S_{M}^{2}+\left(1-\frac{1}{h}\right) S_{E}^{2}+\left\{L_{1}^{2} S_{M}^{4}+\left(1-\frac{1}{h}\right)^{2} L_{2}^{2} S_{E}^{4}+\left(1-\frac{1}{h}\right)^{2} L_{12} S_{M}^{2} S_{E}^{2}\right\}^{\frac{1}{2}}\right]
\end{gathered}
$$

여기서, $\quad F_{1}=F_{\left(\alpha / 2: n_{1}, n_{2}\right)}, \quad F_{2}=F_{\left(1-\alpha / 2: n_{1}, n_{2}\right)}, \quad K_{1}=1-1 / F_{\left(1-\alpha / 2: n_{1}, \infty\right)}, \quad K_{2}=1 / F_{\left(\alpha / 2: n_{2}, \infty\right)}-1$, $K_{12}=\left[\left(F_{2}-1\right)^{2}-K_{1}{ }^{2} F_{2}{ }^{2}-K_{2}{ }^{2}\right] / F_{2}, \quad L_{1}=1 / F_{\left(\alpha / 2: n_{1}, \infty\right)}-1, \quad L_{2}=1-1 / F_{\left(1-\alpha / 2: n_{2}, \infty\right),} L_{12}=\left[\left(1-F_{1}\right)^{2}-L_{1}^{2} F_{1}{ }^{2}-L_{2}^{2} / F_{1}\right.$ 이고 $F_{\left(\delta: n_{1}, n_{2}\right)}$ 는 자유도가 각각 $n_{1}$ 과 $n_{2}$ 인 $F$ 분포의 오른쪽 끝 부분이 $\delta$ 인 $F$ 값을 나타낸다. 그리 고 $\gamma>0$ 이기 때문에 (2.4)로부터 계산된 신뢰하한, 신뢰상한, 또는 신뢰상한과 신뢰하한 모두의 값이 음 일 경우는 그 값을 0으로 한다. (2.4)를 TING 방법이라 하자. 그러나 $\operatorname{Park}(2003$ p.366) 으로부터 총 분산에 대한 급간 분산의 비율로서 급내 상관계수 $\rho=\sigma_{A}^{2} /\left(\sigma_{A}^{2}+\sigma_{E}^{2}\right)$ 가 작은 값을 취 할 때(톡히, $\rho<0.4$ ) TING방법은 신뢰계수가 너무 보수적인(conservative) 신뢰구간을 제공하므 로 적절하지 못하였다.

그러므로 그 대안들로서 $\theta_{M}$ 과 $\theta_{E}$ 의 계수가 양이며 이들의 선형결합으로서 구성된 $\gamma$ 에 대한 신뢰구간을 구할 때, 보다 적절한 Graybill과 Wang(1980) 방법을 사용한 $\gamma$ 에 대한 $100(1-\alpha) \%$ 양쪽 신뢰구간은 다음 식으로부터 구해진다.

$$
\begin{aligned}
& {\left[S_{M}^{2}+\left(1-\frac{1}{h}\right) S_{E}^{2}-\left\{G_{1}^{2} S_{M}^{4}+\left(1-\frac{1}{h}\right)^{2} G_{2}^{2} S_{E}^{4}\right\}^{\frac{1}{2}}\right.} \\
& \left.S_{M}^{2}+\left(1-\frac{1}{h}\right) S_{E}^{2}+\left\{H_{1}^{2} S_{M}^{4}+\left(1-\frac{1}{h}\right)^{2} H_{2}^{2} S_{E}^{4}\right\}^{\frac{1}{2}}\right]
\end{aligned}
$$

여기서, $G_{1}=1-1 / F_{\left(1-\alpha / 2 ; n_{1}, \infty\right)}, G_{2}=1-1 / F_{\left(1-\alpha / 2 ; n_{2}, \infty\right)}, H_{1}=1 / F_{\left(\alpha / 2 ; n_{1}, \infty\right)}-1, H_{2}=1 / F_{\left(\alpha / 2 ; n_{2}, \infty\right)}-1$ 이 고, (2.5)를 GRW 방법이라 하자.

Tsui and Weerahandi(1989)가 제안한 일반화 $p$ 값을 활용한 일반화 축량 방법을 이용한 두 가지 방법을 제안한다. 첫째, Eubank et al.이 보인 (2.2)의 확률분포를 이용하여 $\gamma$ 의 신뢰구간 
90 Dong Joon Park and Soo Jin Lee

을 제안한 Park(2003)의 방법을 정리한다. 둘째, $(2.2 \mathrm{~b})$ 와 같이 근사적인 확률분포를 사용하지 않 고, 정확한 확률분포를 갖는 Olsen et al.이 제안한 통계량을 이용하여 $\gamma$ 의 새로운 신뢰구간을 소개한다. 먼저, Park(2003)이 제안한 $\gamma$ 의 신뢰구간을 다시 정리하면 (2.2a)로부터 $\sigma_{E}^{2}$ 의 추정값 은 $\hat{\sigma}_{E}^{2}=n_{2} s_{E}^{2} / U_{E}^{*}$ 으로 계산되는데 여기서, $s_{E}^{2}$ 은 오차 평균제곱 $S_{E}^{2}$ 의 관찰값들로서 $\sigma_{E}^{2}$ 의 구체적 인 값이 주어진다면 $s_{E}^{2}=\sigma_{E}^{2} \chi_{n_{2}}^{2} / n_{2}$ 으로 계산되고, 일반화 축량 $U_{E}^{*}$ 는 자유도 $n_{2}$ 를 갖고 $\chi^{2}$ 확률 분포를 따르는 확률변수로서 $U_{E}^{*} \sim \chi_{n_{2}}^{2}$ 분포로부터 생성되어진다. 또한 (2.3)의 $\theta_{M}$ 의 추정값은 $\hat{\theta}_{M} \fallingdotseq n_{1} s_{M}^{2} / U_{M}^{*}$ 으로 계산되는데 여기서, $s_{M}^{2}$ 은 $S_{M}^{2}$ 의 관찰값들로서 $\theta_{M}$ 의 구체적인 값이 주어지 면 $s_{M}^{2} \fallingdotseq \theta_{M} \chi_{n_{1}}^{2} / n_{1}$ 으로 계산되고, 일반화 축량 $U_{M}^{*}$ 는 자유도 $n_{1}$ 을 갖고 $\chi^{2}$ 확률분포를 따르는 확 률변수로서 $U_{M}^{*} \sim \chi_{n_{1}}^{2}$ 분포로부터 생성된다. 그러므로 $\gamma$ 의 추정값은 $\theta_{M}$ 와 $\sigma_{E}^{2}$ 에 대한 추정값을 이용하여 $\hat{\gamma}=\hat{\sigma}_{A}^{2}+\hat{\sigma}_{E}^{2}=\hat{\theta}_{M}+(1-1 / h) \hat{\theta}_{E}=n_{1} s_{M}^{2} / U_{M}^{*}+(1-1 / h) n_{2} s_{E}^{2} / U_{E}^{*}$ 로 구해진 다. 이렇게 계산된 $\hat{\gamma}$ 의 값(solution)들로 형성된 확률분포를 $T$ 라고 하면 일반화 축량을 이용한 $\gamma$ 에 대한 $100(1-\alpha) \%$ 양쪽 신뢰구간은 다음 식으로부터 구할 수 있다.

$$
\left[T_{\alpha / 2} \quad ; \quad T_{1-\alpha / 2}\right]
$$

여기서 $T_{\alpha / 2}$ 와 $T_{1-\alpha / 2}$ 는 각각 확률분포 $T$ 의 제 $\alpha / 2$ 백분위수 값과 제 $1-\alpha / 2$ 백분위수 값이다. 식 (2.6)을 GEN1 방법이라 하자.

다음으로 Olsen et al.이 제안한 서로 독립이고 $\chi^{2}$ 확률분포를 따르는 다음의 통계량을 이용한 다.

$$
\frac{Q_{l}}{\left(\sigma_{E}^{2}+d_{l} \sigma_{A}^{2}\right)}=\frac{z^{\prime} E_{l} z}{\left(\sigma_{E}^{2}+d_{l} \sigma_{A}^{2}\right)} \sim \chi_{r_{l}}^{2} ; l=1, \ldots, m
$$

여기서, $E_{l}=e_{l}\left(e^{\prime} e_{l}\right)^{+} e^{\prime}{ }_{l}$ 이고, $e_{l}$ 은 행렬 $W$ 의 고유값 $d_{l}$ 에 대한 고유벡터이며 $S_{E}^{2}, Q_{1}, \ldots, Q_{m}$ 들은 서로 독립임이 보여졌다. 그러므로 $\Sigma Q V /\left(\sigma_{E}^{2}+d_{l} \sigma_{A}^{2}\right)=\chi_{n_{1}}^{2}$ 이 되고, 분모에 $d_{l} \sigma_{E}^{2}$ 을 더하고 뺀 후 정리하면 $\Sigma Q \mathcal{V}\left[d_{l}\left(\gamma-\sigma_{E}^{2}\right)+\sigma_{E}^{2}\right]=\chi_{n_{1}}^{2}$ 로 쓸 수 있고, 일반화 축량 방법을 적용하여

$$
\sum_{l=1}^{m} \frac{q_{l}}{\left[d_{l}\left(\hat{\gamma}-n_{2} s_{E}^{2} / U_{E}^{*}\right)+n_{2} s_{E}^{2} / U_{E}^{*}\right]}=U_{M}^{*}
$$

로 적는다. 이 식에서 $q_{l}$ 은 $Q_{l}$ 의 관찰값들로서 $\sigma_{E}^{2}$ 과 $\sigma_{A}^{2}$ 의 구체적인 값돌이 주어진다면 $q_{l}=\left(\sigma_{E}^{2}+d_{l} \sigma_{A}^{2}\right) \chi_{r_{l}}^{2}$ 로부터 계산되고, 생성된 관찰값 $q_{l}$ 과 관찰값 $s_{E}^{2}$ 와 일반화 축량값 $U_{E}^{*}$ 와 $U_{M}^{*}$ 를 (2.8)에 대입하여 계산한 $\hat{\gamma}$ 의 값들로 형성된 확률분포를 $V$ 라고 하자. 그러면 일반화 축량을 이용한 $\gamma$ 에 대한 $100(1-\alpha) \%$ 양쪽 신뢰구간은 다음 식으로부터 구할 수 있다. 


$$
\left[\begin{array}{lll}
V_{\alpha / 2} & ; & V_{1-\alpha / 2}
\end{array}\right]
$$

여기서 $V_{\alpha / 2}$ 와 $V_{1-\alpha / 2}$ 는 각각 확률분포 $V$ 의 제 $\alpha / 2$ 백분위수 값과 제 $1-\alpha / 2$ 백분위수 값이다. 식 (2.9)를 GEN2 방법이라고 하자.

\section{3. 모의실험}

무수히 많고 다양한 불균형 설계 가운데서 El-Bassiouni and Seely(1996)는 일곱 가지의 설계 를 이용하여 신뢰구간에 관한 추론을 하였는데, 그 설계의 불균형 측도(measure of imbalance) 는 0.253 에서 0.887 이었다. 여기서 불균형 측도란 Ahrens와 Pincus(1981)가 제안한 것으로서 $J_{i}$ 의 크기들의 산술평균에 대한 조화평균의 비율 $\left(\Sigma_{i=1}^{I} J_{i}^{-1} / I\right)^{-1} /\left(\Sigma_{i=1}^{I} J_{i} / I\right)$ 을 의미한다. 보다 더 불균형 상태가 심각한 값에 대해서도 $\gamma$ 에 대한 신뢰구간이 적절한가를 알아보기 위하여 세 가 지. 설계(<표 3.1>의 설계번호 $6,9,10$ )를 더 추가하여 불균형 측도가 0.08 에서 0.887 이 되는 모 두 10 가지 유형에 대한 $\gamma$ 의 신뢰구간을 비교한다. <표 3.1 >에는 모의실험에 사용된 10 가지 유 형과 군의 수 $I$ 와 군의 크가 $J_{i}$ 와 불균형 측도를 포함한다. 모의실험의 실행을 위하여 $\rho=\sigma_{A}^{2} /\left(\sigma_{A}^{2}+\sigma_{E}^{2}\right)$ 에서 일반성을 잃지 않고 $\sigma_{A}^{2}=1-\sigma_{E}^{2}$ 으로 쓸 수 있고, 따라서 $\rho=\sigma_{A}^{2}$ 과 $1-\rho=\sigma_{E}^{2}$ 으로 쓴다. $\rho=0.001,0.1,0.2,0.3,0.4,0.5,0.6,0.7,0.8,0.9,0.999$ 로 모두 11 개의 $\rho$ 값에 대하여 2000 번씩 모의실험을 실행시켜서 $\gamma$ 의 신뢰구간을 계산한다.

<표 3.1> 모의실험에 사용된 불균형 설계

\begin{tabular}{c|c|c|c|c}
\hline 설계 & 표본의 크기 & $I$ (군의 수) & $J_{i}$ (군의 크기) & 불균형 측도 \\
\hline 1 & 15 & 3 & $3,5,7$ & 0.887 \\
2 & 15 & 3 & $1,5,9$ & 0.458 \\
3 & 30 & 3 & $2,10,18$ & 0.458 \\
4 & 30 & 6 & $1,1,5,5,9,9$ & 0.458 \\
5 & 30 & 6 & $1,1,1,1,13,13$ & 0.289 \\
6 & 157 & 6 & $1,1,2,3,50,100$ & 0.080 \\
7 & 45 & 9 & $1,1,1,5,5,5,9,9,9$ & 0.458 \\
8 & 45 & 9 & $1,1,1,1,1,1,1,19,19$ & 0.253 \\
9 & 59 & 10 & $1,1,4,5,6,6,8,8,10,10$ & 0.524 \\
10 & 55 & 10 & $1,2,3,4,5,6,7,8,9,10$ & 0.621 \\
\hline
\end{tabular}

<그림 3.1 >과 <그림 3.2 >와 <그림 3.3>과 <그림 3.4 >는 각각 $\gamma$ 의 명시된 신뢰계수가 $90 \%$ 와 $95 \%$ 일 때 앞에서 언급한 $\rho$ 의 11 개의 값에 대하여 모의실험에 의하여 구한 신뢰구간들의 신 뢰계수와 신뢰구간들의 평균길이를 상자그림으로 나타낸 것이다. 모의실험의 수행정도를 분석하 기 위하여 이항분포에 대한 정규근사를 사용하면 $\gamma$ 의 참 신뢰계수가 0.90(0.95)일 때 2000희 반 복 모의실험 결과에 따른 추정된 신뢰계수가 $0.8866(0.9404)$ 보다 작을 기회는 $2.5 \%$ 보다 더 작다. 
92 Dong Joon Park and Soo Jin Lee
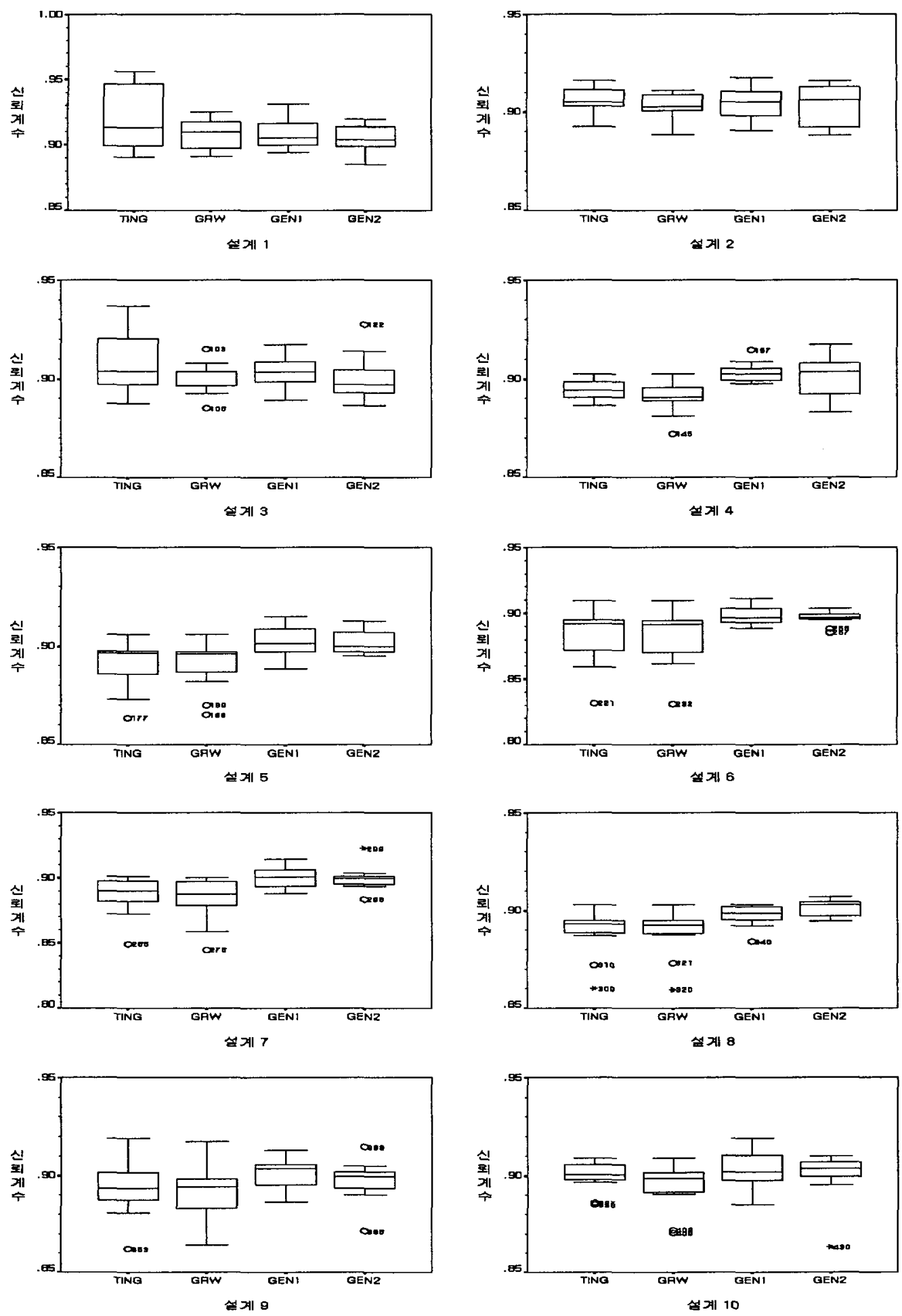

<그림 $3.1>\gamma$ 의 명시된 신뢰계수가 $90 \%$ 일 때 모의실험으로부터 구한 신뢰계수 
Confidence Intervals on Variance Components 93
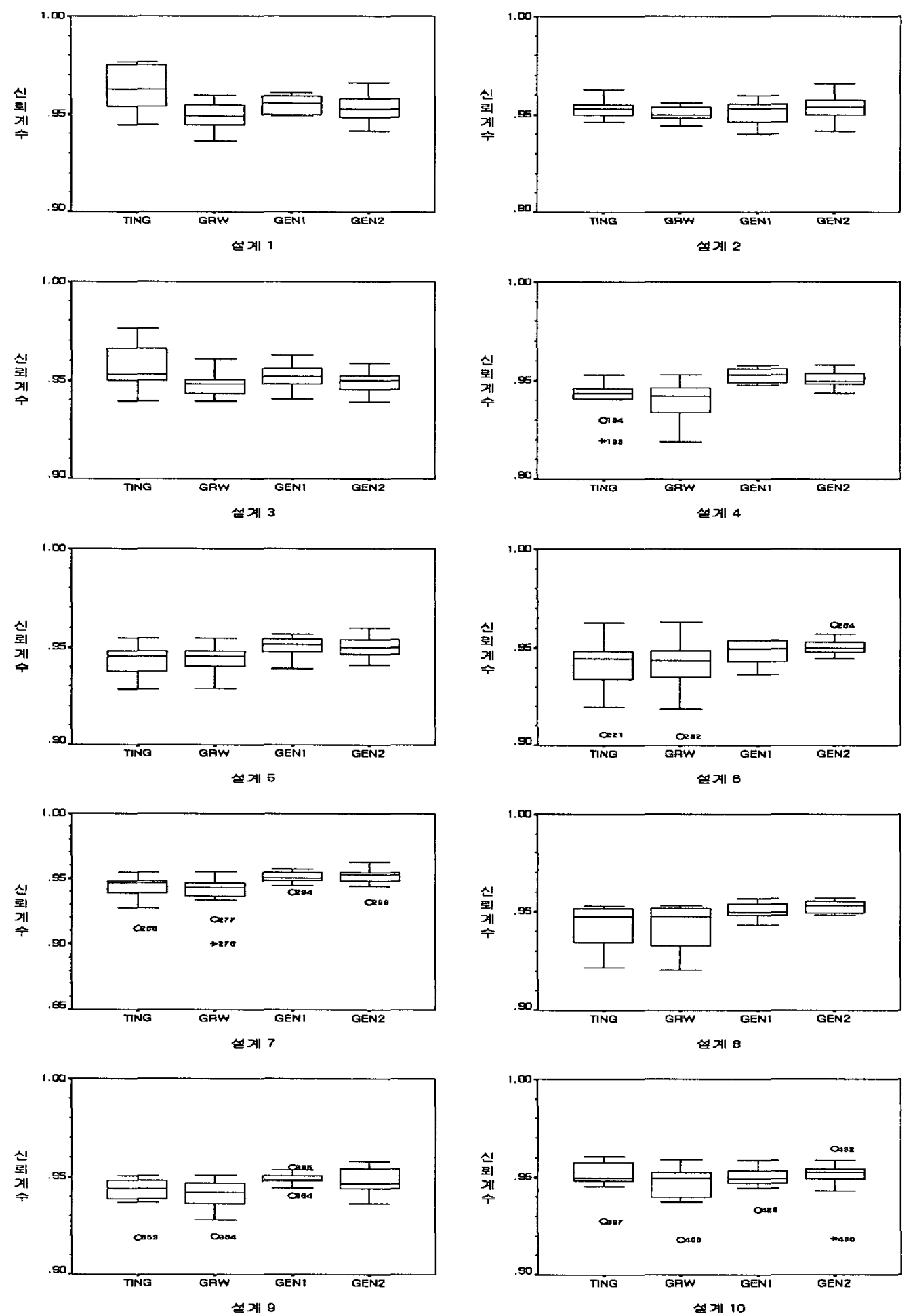

<그림 3.2> $\gamma$ 의 명시된 신뢰계수가 $95 \%$ 일 때 모의실험으로부터 구한 신뢰계수 
94 Dong Joon Park and Soo Jin Lee
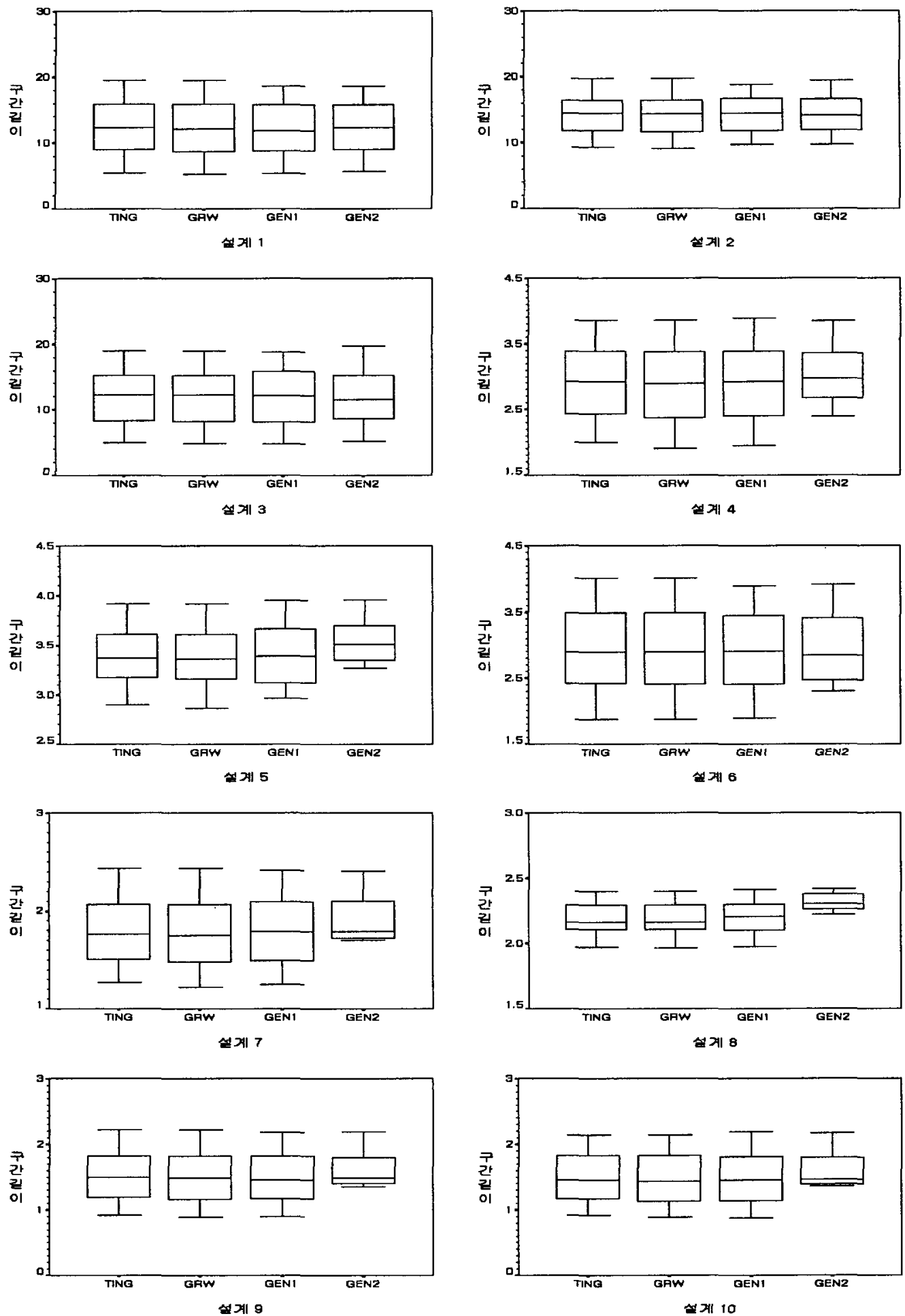

<그림 3.3> $\gamma$ 의 명시된 신뢰계수가 $90 \%$ 일 때 신뢰구간의 평균길이 
Confidence Intervals on Variance Components 95
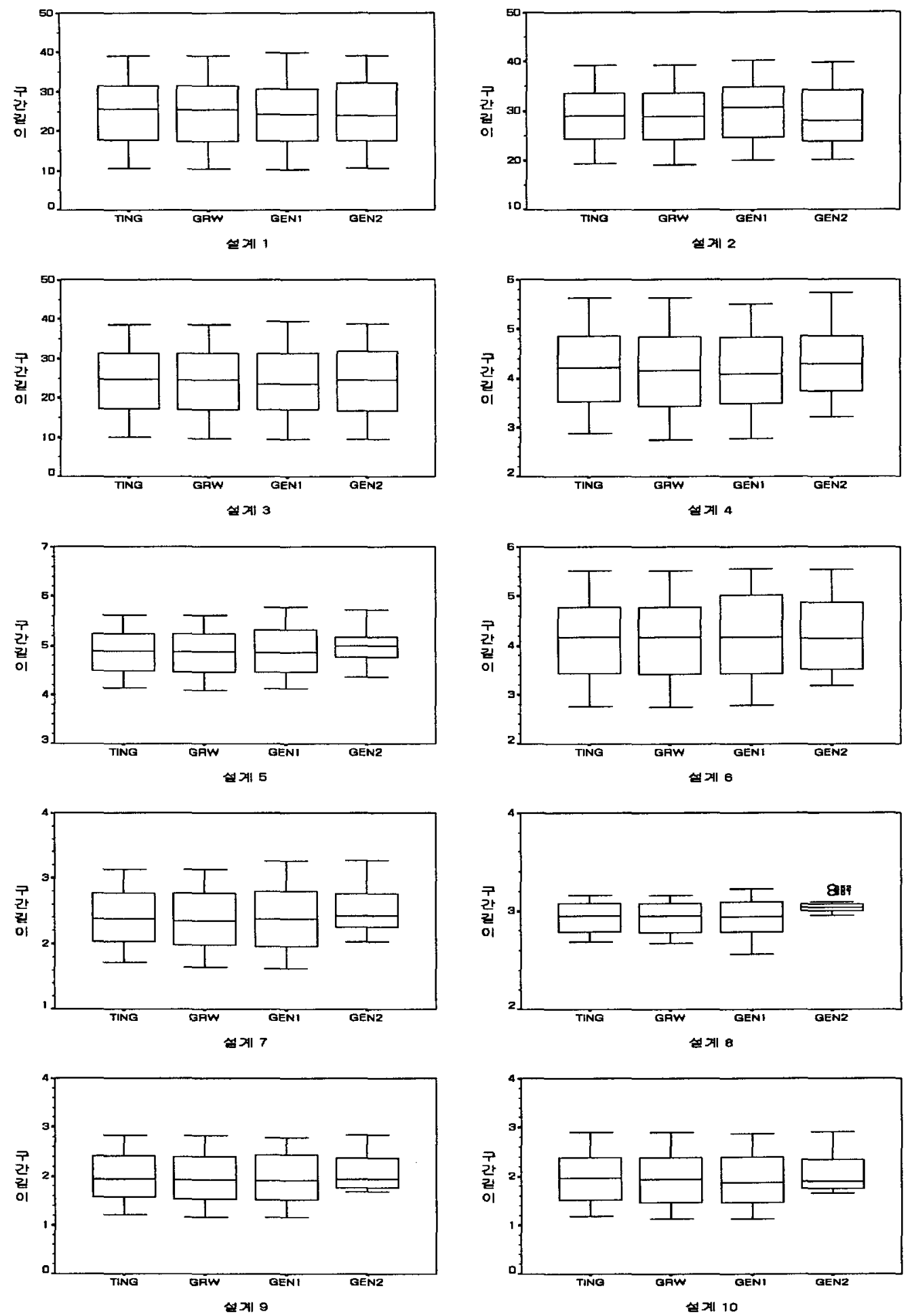

<그림 3.4> $\gamma$ 의 명시된 신뢰계수가 $95 \%$ 일 때 신뢰구간의 평균길이 
96 Dong Joon Park and Soo Jin Lee

<그림 3.1>로부터 군 $(I)$ 의 수가 3 인 설계번호 $1,2,3$ 의 경우, 네 가지 방법 모두가 대체로 신 뢰계수를 유지하고 있으나, TING방법은 군의 크기 $\left(J_{i}\right)$ 의 변화가 작을 때와 클 때 모의실험으로 부터 구한 신뢰계수가 명시된 신뢰계수를 훨씬 능가하는 보수적인 결과를 보여준다. 즉, 설계번 호 2 의 경우는 비교적 적절한 신뢰계수를 나타내지만, 설계번호 1 의 경우 $\rho<0.4$ 일 때와 설계 번호 3 의 경우 $\rho<0.3$ 일 때 지나치게 보수적인 결과를 보여준다. $\gamma$ 의 명시된 신뢰계수가 $95 \%$ 일 때인 <그림 $3.2>$ 의 경우도 이와 유사한 양상을 보인다. 즉, TING방법은 설계번호 1 과 3 의 경우 각각 $\rho<0.4$ 일 때와 $\rho<0.3$ 일 때 지나치게 보수적인 신뢰계수가 나타난다.

그러나 <그립 3.1 >과 <그림 3.2 >로부터 군의 수가 중가 $(I \geqq 6)$ 하거나, 불균형 측도의 변화가 심하거나, 군의 크기 $\left(J_{i}\right)$ 가 1 에서부터 100 까지 급격히 변하거나, 1 부터 10 까지 점차적으로 변하 는 경우에는 $\rho>0.3$ 일 때 TING방법과 GRW방법이 모의실험을 통해 구한 신뢰계수가 명시된 신뢰계수를 유지하지만, 대략적으로 $\rho<0.3$ 일 때는 두 방법 모두 모의실험으로 구한 신뢰계수 가 명시된 신뢰계수(모의실험을 통해 구한 신뢰계수가 0.8866(0.9404)보다 작음)보다 작은 값들 이 나타남을 볼 수 있다. 구체적으로 설명하면 <그림 3.1>에서 설계번호 4의 경우 TING방법의 $\rho<0.2$ 일 때, GRW방법의 $\rho<0.3$ 일 때, 설계번호 $5,7,9$ 의 경우 TING방법과 GRW방법의 $\rho<0.3$ 일 때, 설계번호 6 의 경우 TING방법과 GRW방법의 $\rho<0.4$ 일 때, 설계번호 8 과 10 의 경우 TING방법과 GRW방법의 $\rho<0.2$ 일 때 명시된 신뢰계수가 유지하지 못하고 있고 <그림 $3.2>$ 에서도 이와 유사한 경향을 보인다.

<표 3.2> $\gamma$ 의 명시된 신뢰계수가 $90 \%$ 일 때 모의실험 결과

\begin{tabular}{c|cccc|cccc}
\hline 5번 설계 & \multicolumn{3}{|c}{ 모의실험으로 구한 신뢰구간의 신뢰계수 } & \multicolumn{4}{|c}{ 모의실험으로 구한 신뢰구간의 평균길이 } \\
\hline$\rho$ & TING & GRW & GEN1 & GEN2 & TING & GRW & GEN1 & GEN2 \\
\hline 0.001 & 0.8635 & 0.8650 & 0.9015 & 0.8960 & 2.8946 & 2.8639 & 2.9645 & 3.3057 \\
0.1 & 0.8730 & 0.8700 & 0.8980 & 0.9125 & 3.0266 & 3.0026 & 3.0404 & 3.2698 \\
0.2 & 0.8795 & 0.8820 & 0.9125 & 0.9120 & 3.1147 & 3.0956 & 3.0711 & 3.3575 \\
0.3 & 0.8975 & 0.8965 & 0.9015 & 0.8970 & 3.2355 & 3.2220 & 3.1618 & 3.3372 \\
0.4 & 0.8935 & 0.8930 & 0.8950 & 0.8975 & 3.3086 & 3.2983 & 3.2520 & 3.5232 \\
0.5 & 0.8965 & 0.8960 & 0.9005 & 0.8985 & 3.3708 & 3.3643 & 3.3874 & 3.4271 \\
0.6 & 0.8920 & 0.8915 & 0.9085 & 0.9000 & 3.4456 & 3.4419 & 3.4434 & 3.5089 \\
0.7 & 0.9060 & 0.9060 & 0.8885 & 0.8950 & 3.5331 & 3.5320 & 3.6408 & 3.7468 \\
0.8 & 0.8975 & 0.8975 & 0.9150 & 0.9015 & 3.6903 & 3.6903 & 3.6926 & 3.6439 \\
0.9 & 0.9030 & 0.9030 & 0.8965 & 0.9105 & 3.8462 & 3.8467 & 3.7549 & 3.7856 \\
0.999 & 0.8970 & 0.8970 & 0.9090 & 0.9030 & 3.9177 & 3.9177 & 3.9533 & 3.9624 \\
\hline MAX & 0.9060 & 0.9060 & 0.9150 & 0.9125 & 3.9177 & 3.9177 & 3.9533 & 3.9624 \\
MIN & 0.8635 & 0.8650 & 0.8885 & 0.8950 & 2.8946 & 2.8639 & 2.9645 & 3.3057 \\
\hline
\end{tabular}

<그립 3.3>과 <그림 3.4 >로부터 신뢰구간의 평균길이는 군의 수가 3 일 때, 군의 크기의 변화 에 관계없이 설계번호 1 부터 3 까지의 경우 네 가지 방법 모두가 근소한 차이를 보이고 있고, $\rho$ 가 증가함에 따라 신뢰구간은 증가한다. 군의 수가 증가 $(I \geqq 6)$ 하거나, 군의 크기의 변화가 심한 
설계번호 4 부터 10 까지의 경우, $\rho$ 가 작을 때(약 $\rho<0.3$ ) 신뢰계수를 유지하지 못하는 TING방 법과 GRW방법을 제외한 GEN1방법과 GEN2방법의 신뢰구간의 평균길이들을 비교하면 근소한 차이를 보이고 있지만 GEN1방법이 GEN2방법보다 약간 짦은 신뢰구간을 제공한다.

$\rho$ 가 클 때(약 $\rho>0.3$ )는 네가지 방법 모두 비슷한 평균길이를 갖는 신뢰구간을 제공한다. 특 히, GEN2방법은 군의 수가 6 이상인 설계번호 4 부터 10 까지 군의 크기의 다양한 변화에 관계없 이 $\rho$ 의 모든 값에 대하여 신뢰구간의 평균길이의 변화가 가장 작음을 보여준다.

\section{4. 예제의 적용}

$\gamma$ 의 네 가지 신뢰구간을 계산하기 위하여 Milliken과 Johnson(2002, p.428 429)의 수학을 가 르치는 세 가지 방법들을 평가하기 위한 한 교육청의 연구자료를 사용한다. 이 자료를 이용하여 모형 (1.1)에 나타난 $\gamma$ 의 신뢰구간을 계산하기 위하여 사전점수를 설명변수 $X_{i j}$, 성적을 반응변 수 $Y_{i j}$, 구체적인 교육방법으로 가르치는 교사들의 효과를 $A_{i}$, 그리고 학생 개인에 따른 오차를 $E_{i j}$ 로 가정한다. <표 3.1 >의 설계번호 5 는 군의 크기가 각각 $1,1,1,1,13,13$ 이므로 8년의 경력 을 가진 7 번째 교사와 6 년의 경력을 가진 9 번째 교사들의 학생 13 명을 모두 선택하여 $J_{5}=J_{6}=13$ 으로 하고, 나머지 7 명의 교사들 가운데 4 명의 교사를 랜덤으로 선택한 결과 2 번째, 4 번째, 5 번째, 8 번째 교사가 선택되었다. 그리고 $J_{1}=1, J_{2}=1, J_{3}=1, J_{4}=1$ 이므로 각 선택된 교사들이 가르치는 학생들을 대상으로 하여 한 명씩 랜덤으로 선택한 결과 각각 8 번째, 2 번째, 8 번 째, 9 번째 학생들이 선택되었고 그 자료들을 <표 4.1>에 수록하였다.

<표 4.1> Milliken과 Johnson(2002)에서 랜덤으로 선택된 자료들

\begin{tabular}{|c|c|c|c|c|c|c|c|c|c|c|c|c|}
\hline \multirow[b]{3}{*}{$\begin{array}{l}\text { 관 } \\
\text { 찰 } \\
\text { 값 }\end{array}$} & \multicolumn{12}{|c|}{ 교 사 } \\
\hline & \multicolumn{2}{|c|}{2} & \multicolumn{2}{|c|}{4} & \multicolumn{2}{|c|}{5} & \multicolumn{2}{|c|}{8} & \multicolumn{2}{|c|}{7} & \multicolumn{2}{|c|}{9} \\
\hline & $\begin{array}{c}\text { Score } \\
\text { (Y) }\end{array}$ & $\begin{array}{l}\text { Pre- } \\
\text { score } \\
(\mathrm{X})\end{array}$ & $\begin{array}{c}\text { Score } \\
\text { (Y) }\end{array}$ & $\begin{array}{l}\text { Pre- } \\
\text { score } \\
(\mathrm{X})\end{array}$ & $\begin{array}{c}\text { Score } \\
\text { (Y) }\end{array}$ & $\begin{array}{l}\text { Pre- } \\
\text { score } \\
\text { (X) }\end{array}$ & $\begin{array}{c}\text { Score } \\
\text { (Y) }\end{array}$ & $\begin{array}{l}\text { Pre- } \\
\text { score } \\
(\mathrm{X})\end{array}$ & $\begin{array}{c}\text { Score } \\
(\mathrm{Y})\end{array}$ & $\begin{array}{l}\text { Pre- } \\
\text { score } \\
(\mathrm{X})\end{array}$ & $\begin{array}{c}\text { Score } \\
\text { (Y) }\end{array}$ & $\begin{array}{l}\text { Pre- } \\
\text { score } \\
(X)\end{array}$ \\
\hline 1 & 79 & 62 & 85 & 67 & 85 & 68 & 90 & 92 & 89 & 93 & 99 & 88 \\
\hline 2 & & & & & & & & & 94 & 75 & 99 & 89 \\
\hline 3 & & & & & & & & & 85 & 75 & 89 & 78 \\
\hline 4 & & & & & & & & & 84 & 72 & 95 & 75 \\
\hline 5 & & & & & & & & & 85 & 63 & 90 & 76 \\
\hline 6 & & & & & & & & & 84 & 65 & 91 & 82 \\
\hline 7 & & & & & & & & & 91 & 78 & 92 & 90 \\
\hline 8 & & & & & & & & & 88 & 73 & 92 & 74 \\
\hline 9 & & & & & & & & & 88 & 69 & 92 & 75 \\
\hline 10 & & & & & & & & & 89 & 68 & 90 & 70 \\
\hline 11 & & & & & & & & & 88 & 62 & 94 & 92 \\
\hline 12 & & & & & & & & & 84 & 66 & 90 & 78 \\
\hline 13 & & & & & & & & & 83 & 66 & 92 & 89 \\
\hline
\end{tabular}

<표 4.1>의 자료에 대한 SAS의 PROC MIXED의 REML(Restricted Maximum Likelihood)을 
98 Dong Joon Park and Soo Jin Lee

이용한 교사들의 효과에 대한 분산 $\left(\sigma_{A}^{2}\right)$ 과 학생 개인에 따른 오차의 분산 $\left(\sigma_{E}^{2}\right)$ 의 추정값을 계산하 기 위하여 <그림 4.1 >의 SAS 프로그램의 실행결과 각각 $\hat{\sigma}_{A}^{2}=6.3177, \hat{\sigma}_{E}^{2}=8.2789$ 으로 계산 되었다.

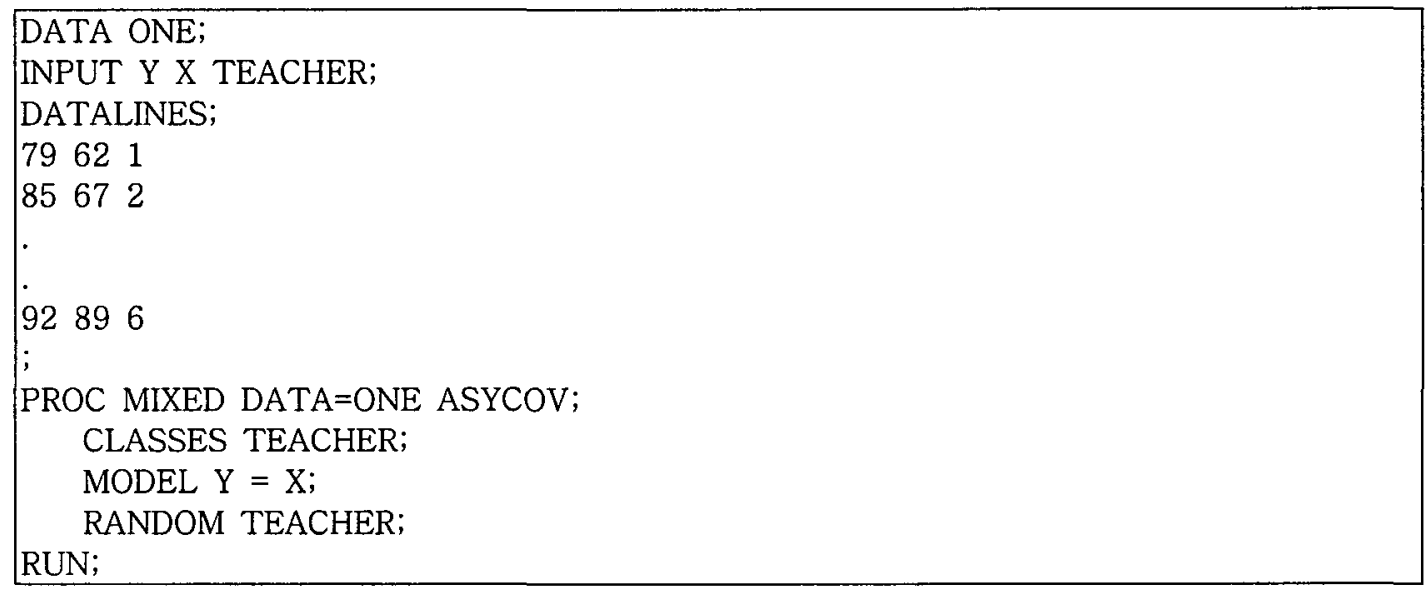

<그림 4.1> 분산들의 추정값을 계산하기 위한 SAS 프로그램

주어진 자료를 이용하여 TING, GRW, GEN1, GEN2 방법으로 교사들의 효과에 대한 분산 $\left(\sigma_{A}^{2}\right)$ 과 학생 개인에 따른 오차의 분산 $\left(\sigma_{E}^{2}\right)$ 의 합 $\gamma$ 에 대한 $90 \%$ 신뢰구간을 계산한 결과는 <표 $4.2>$ 와 같다. 급내 상관계수의 추정량은 $\hat{\rho}=\hat{\sigma}_{A}^{2} /\left(\hat{\sigma}_{A}^{2}+\hat{\sigma}_{E}^{2}\right)=0.4328$ 으로서 <그림 $3.1>$ 과 <그림 3.2>와 <표 3.2>로부터 설계번호 5 에 대한 모의실헙으로 구한 신뢰계수가 $0.4<\rho<0.5$ 일 때는 명시된 신뢰계수 0.9 에 매우 가까운 값을 제시하므로 $\gamma$ 에 대한 $90 \%$ 신뢰구간을 계산하 기 위해서는 TING, GRW, GEN1, GEN2 방법 모두 사용 가능하다.

<표 $4.2>\gamma$ 의 $90 \%$ 신뢰구간

\begin{tabular}{cccc}
\hline 방법 & 신뢰하한 & 신뢰상한 & 구간의 길이 \\
\hline TING & 6.21 & 45.41 & 39.20 \\
\hline GRW & 6.37 & 45.52 & 39.15 \\
\hline GEN1 & 6.46 & 44.81 & 38.35 \\
\hline GEN2 & 7.61 & 45.99 & 38.38 \\
\hline
\end{tabular}

위에서 구한 $\gamma$ 의 신뢰구간은 모형 (1.1)을 만족하는 것으로 가정하고 계산된 값들이다. 만약 군과 관련된 오차항 $A_{i}$ 와 군 내부의 개별자료에 대한 오차항 $E_{i j}$ 가 정규분포의 가정을 만족하지 않을 때는 앞의 네 가지 방법들을 사용할 수 없고, 비정규오차항을 포함하는 흔합모형에서 분산 들의 추정과 함께, 정규분포 가정의 위배에 대한 robustness에 대한 문제는 광범위한 문헌연구 와 Searle et al.(1992), Hocking(2003), Cox와 Solomon(2003)등의 문헌을 통하여 다음 연구과제 에서 다루겠다. 


\section{5. 결론}

지금까지 불균형 중첩 오차구조를 갖는 단순 선형 회거모형에서 나타나는 두 분산 $\sigma_{A}^{2}$ 과 $\sigma_{E}^{2}$ 의 합 $\gamma=\sigma_{A}^{2}+\sigma_{E}^{2}$ 에 대한 신뢰구간을 구하는 TING방법과 GEN1방법 외에 그 대안으로서 GRW방 법과 GEN2방법을 유도하였다. 요약하면 군의 수가 $I=3$ 일 때는 네 가지 방법 모두가 신뢰계수 를 유지하였지만 TING방법은 설계번호 1 과 3 에서 $\rho$ 의 값이 작게 나타날 때 신뢰계수가 지나치 게 보수적인 결과를 보여주므로 바람직하지 않고 GRW방법과 GEN1방법과 GEN2방법을 사용하 여 신뢰구간을 계산한 다음, 신뢰구간이 가장 짧게 나타나는 방법을 추천한다. 그러나 군의 수 가 $I \geqq 6$ 이고 $\rho \geqq 0.3$ 일 때는 GRW방법이나 비교적 정확한 신뢰계수를 유지하는 GEN1방법 또 는 GEN2방법을 이용하여 신뢰구간이 짧은 것을 선택할 수 있지만 $I \geqq 6$ 이고 약 $\rho<0.3$ 일 때 는 TING방법과 GRW방법은 신뢰계수를 유지하지 못하므로 사용할 수 없고, GEN1방법과 $\mathrm{GEN} 2$ 방법을 사용해서 신뢰구간을 계산한 다음, 가장 짧은 신뢰구간을 나타내는 방법을 사용할 것을 제안한다.

\section{참고문헌}

[1] Ahrens, H. and Pincus, R. (1981), On two measures of unbalancedness in a one-way model and their relation to efficiency, Biometrical Journal, 23, 227-235.

[2] Burdick, R. K. and Graybill, F. A. (1992), Confidence Intervals on Variance Components, Marcel Dekker.

[3] Cox, D. R. and Solomon, P. J. (2003), Components of Variance, Chapman \& Hall/CRC.

[4] El-Bassiouni, M. Y. (1994), Short confidence intervals for variance components, Communications in Statistics Theory and Method, 23(7), 1915-1933.

[5] El-Bassiouni, M. Y. and Seely, J. F. (1996), A modified harmonic mean test procedure for variance components, Journal of Statistical Planning and Inference, 49, $319-326$.

[6] Eubank, L., Seely, J., and Lee, Y. (2001), Unweighted mean squares for the general two variance component mixed model, Proceeding of the Graybill Conference, Ft. Collins, Co., June, 281-290.

[7] Graybill, F. A. and Wang, C-M. (1980), Confidence intervals on nonnegative linear combinations of variances, Journal of the American Statistical Association, 75, 869-873.

[8] Hocking, R. R. (2003), Methods and Applications of Linear Models, John Wiley \& Sons, Inc.

[9] Milliken, G. A. and Johnson, D. E. (2002), Analysis of Messy Data Voume II : Analysis of Covariance, Chapman \& Hall/CRC.

[10] Olsen, A., Seely, J., and Birkes, D. (1976), Invariant Quadratic Unbiased Estimation for 
100 Dong Joon Park and Soo Jin Lee

Two Variance Components, Annals of Statistics, 4, 878-890.

[11] Park, D. J. (2003), Interval estimation for sum of variance components in a simple linear regression model with unbalanced nested error structure, The Korean Communications in Statistics, 10(2), 361-370.

[12] Park, D. J. and Burdick, R. K. (2003), Performance of confidence intervals in regression models with unbalanced one-fold nested error structure, Communications in Statistics Simulation and Computation, 32(3), 717-732.

[13] Satterthwaite, F. E. (1941), Synthesis of Variance, Psychometrika, 6, 309-316.

[14] Satterthwaite, F. E. (1946), An Approximate Distribution of Estimates of Variance Components, Biometrics Bulletin, 2, 110-114.

[15] Searle, S. R., Casella, G., and McCulloch, C. E. (1992), Variance Components, John Wiley \& Sons, Inc.

[16] Smith, H. F. (1936), The Problem of Comparing the Results of Two Experiments with Unequal Errors, Journal of the Council of Scientific and Industrial Research, 9, 211-212.

[17] Ting, N., Burdick, R.K., Graybill, F. A., Jeyaratnam, S., and Lu, T.-F. C.(1990), Confidence Intervals on Linear Combinations of Variance Components, Journal of Statistical Computation and Simulation, 35, 135-143.

[18] Tsui, K and Weerahandi, S. (1989), Generalized p-value in significance testing of hypotheses in the presence of nuisance parameters, Journal of American Statistical Association, 84, 602-607. 